\title{
Graphene sensor for smart phone based continuous monitoring of ECG signals
}

\author{
Mohammed Butt ${ }^{1}$, Maysam Abbod ${ }^{1}$ and Antti Vehkaoja ${ }^{2}$ and Wamadeva Balachandran ${ }^{1 *}$ \\ ${ }^{1}$ Department of Electronic and Computer Engineering, Brunel University London, Uxbridge, UB8 3PH, UK \\ ${ }^{2}$ Faculty of Medicine and Health Technology, Tampere University, Tampere, Finland
}

\begin{abstract}
ECG (Electrocardiography) is an important vital sign in the body of a human, which can detect different illness including cardiac arrhythmias, heart attacks and cardiomyopathy. Continuous monitoring of ECG can prevent such type of diseases on time and help to control the death rates and decrease the healthcare costs by enabling early intervention. In this regard, this paper presents a graphene-based ECG sensor for continuous monitoring using behind the ear location. Due to excellent electrical conductivity, the graphene layer is coated on the surface of $\mathrm{Ag} / \mathrm{AgCl} \mathrm{ECG} \mathrm{measurement} \mathrm{electrodes} \mathrm{using} \mathrm{a} \mathrm{drop} \mathrm{casting} \mathrm{technique} \mathrm{for} \mathrm{the}$ improvement of skin to electrode contact. The quality of the contact is further compared with traditional $\mathrm{Ag} / \mathrm{AgCl} \mathrm{electrodes}$ to evaluate the performance in terms of the better output signal. The obtained results showed that the signal from graphene-based electrodes is better than the one obtained with conventional electrodes in term of SNR (signal to noise ratio) and shoes that all the waveforms of a cardiac cycle very clearly. Furthermore, the ECG acquisition unit is integrated with the CBT (Core body temperature) sensor capable of measuring temperature from the tympanic membrane (in the ear). Both sensors are capable of measuring the vital signs from the ear location. Moreover, the device is capable of sending the data to the mobile application via Bluetooth communication, the application running in the phone of the user would allow continuous monitoring of their ECG and CBT in real time by providing a user-friendly environment.
\end{abstract}

\section{Introduction}

The new era of the world has the fastest growth in the field of technology related to embedded systems and wireless communications. Where the technology is provided with large applications in the daily life of people in different fields, it also provides the excellent quality of services in the field of health care. The e-health has arisen in the last few years with the concept of technology, communication and medical treatment together, which provides the platform to the people for monitoring their health condition 24/7 in their smart devices. In this regard, $\mathrm{m}$-health is one of the latest technologies with the main aim to provide the health care facilities to users around the world on their mobile phones and other communication devices. Mobile health has arisen by means of the advancement in mobile communication and wireless technology. There are 1 billion smartphones and 100 million tablets around the world which can be used in health monitoring purposes to control the spread of infectious diseases and to minimize untimely deaths due to heart diseases [1].

Cardiovascular diseases remain the leading cause of death globally. Nearly 17.3 million people die of this every year around the world, and it is expected that the rate will grow to more than 23.6 million by 2030 . The population living in low and middle -income countries are normally more affected by cardiovascular diseases. Usually, $80 \%$ of deaths occur in younger ages in these countries [2]. The concept of $m$-health is to provide continuous monitoring of the physiological parameters in order to control the rising death rate due to cardiovascular diseases. The continuous monitoring of HR can help to identify the patient risk for different diseases such as heart attack, heart failure, stroke and renal failure.

The wearable integrated system, able to bring together the different vital sign such as ECG and CBT in a small device that is proposed.
The monitoring system also brings together the concept of Wireless Wide Band Network (WBAN) and Wireless Personal Area Network (WPAN). Different medical sensors which are located on different areas of the body allow to send the collective data to the server and to send the data to smartphones, tablets and other devices via different wireless technologies such as Bluetooth, ZigBee, and 3G [3].

The main aim of the proposed system is to design the easily wearable device which can monitor the health condition of the person and also display the required parameter on the smart devices in real time which would allow the patient to monitor the health condition in the user-friendly environment. The proposed sensors will be located in and behind the ear; the ECG signals will be recorded using the sensors located behind the ear while the Core Body Temperature (CBT) sensor will be inserted into the ear canal. Behind the ear concept is proposed in order to avoid removing the apparels to locate the sensors in the torso area. The data from both sensors can be gathered using the App, which is installed on smart devices of users with the help of wireless communication technologies. Furthermore, in order to improve the signal-to-noise ratio, graphene coated ECG sensors were used to improve the capacitive coupling between the ECG sensors and skin due to its good electrical and physical properties hence enhancing the ECG signal quality.

${ }^{*}$ Correspondence to: Wamadeva Balachandran, Department of Electronic and Computer Engineering, Brunel University London, Uxbridge, UB8 3PH, UK, Tel: +44 (0) 1895 265774; Fax: +44 (0) 1895 269782; E-mail: wamadeva. balachandran@brunel.ac.uk

Key words: graphene-based sensor, ECG, smart phones

Received: March 07, 2019; Accepted: March 21, 2019; Published: March 25 2019 


\section{Literature review}

Different health monitoring system have been designed which are capable of monitoring different vital signs mainly ECG, PPG, CBT and many others which can also provide remote access to body sensors using different wireless communication techniques.

He et al. [4] presented a wearable vital sign monitoring system on the ear which can measure different vital signs such as ECG, BCG (Bacillus Calmette Guerin) and PPG (photoplethysmography) with the help of commercially available sensors. In their proposed work they also demonstrated the ear is a natural anchoring point for integrated sensing of different body signs. Further developments were done by Lee et al. [5] by designing a system which can take the blood glucose level and ECG signals from the human body. Their main purpose was to investigate the efficiency of $\mathrm{m}$-health monitoring service using Zigbee device. On the other hand, wearable (PPG) sensor module which is based on PSoC (programmable system-on-chip) can monitor a sudden health issue of a driver that could cause major road accidents. This system records the driver's PPG value and sends data to the patient's mobile phone via Bluetooth technology, which can be linked to the emergency services, Lin et al. [6]. Mundt et al. [7] designed a wearable ambulatory physiological sign monitoring system for space and terrestrial applications. It was named "Life Guard". The system can measure different vital sign such as three lead ECG, respiration rate with the help of impedance plethysmography, heart rate, $\mathrm{SpO}_{2}, \mathrm{CBT}$ and Blood Pressure (BP). Vesico et al. [8] presented work related to the measurement of Heart Rate Variability (HRV) using PPG sensor, which is clipped on the ear lobe. Poh et al. [9] proposed a PPG measuring ear worn device called 'Heart Phone' to monitor the condition of the heart. The system involves a PPG measurement sensor embedded in the earbud of regularly available earphones, which can easily be worn in the ear.

Recent studies have illustrated that graphene (GN) based sensor can bring changes in the world future industry. Furthermore, GN material has contributed to improving the sensitivity of the sensors and especially biosensors because of its unique electrochemical and physical properties. Graphene plays an important role in order to improve the performance of sensors especially in clinical, environmental and food research applications [10]. In this study the $\mathrm{Ag} / \mathrm{AgCl} \mathrm{ECG} \mathrm{electrodes}$ and conventional thermopile-based CBT sensor surfaces were coated with grapheme using drop casting techniques. The finished surfaces were not homogeneously covered with grapheme; about $80 \%$ of the surfaces were coved with grapheme.

\section{Graphene sensor technology}

Graphene is a 2-dimensional element which is made of carbon atoms. It has some outstanding properties which give them priority over other elements. One of the most important properties of graphene is its electrical conductivity which has increased its use in the world of electronics, especially in sensor technology.

Graphene is an element of carbon atom group with zero-overlap of semimetals. It contains both holes and electrons as a charge carrier in the outer shell. The structure of graphene is 2-dimensional with four electrons present in the outer shell for bonding, three of them relate to another carbon atom, and one electron is freely available in the third dimension for electronic conduction. The second important property of graphene is its mechanical strength; the graphene is also the strongest material in the world due to the narrow size bonding between the two carbon atoms of its structure which is $0.142 \mathrm{Nm}$-long.
It is also very light in weight of about $0.77 \mathrm{mg} / \mathrm{m}^{2}$. Graphene material also shows some optical properties such as able to absorb $2.3 \%$ of white light because of its good electronic properties [11]. It is highly sensitive to an infrared source; hence this property is utilized in the CBT sensor.

Due to the versatile electrical and physical properties of graphene, the graphene plays a significant role in the field of sensors, especially in biosensors world. Justino et al. [12] presented the work related to the role of graphene in sensing technology especially the contribution in biosensors. Most of the reported work in the last few years is related to graphene materials (GO, rGO). The performance of graphene-based sensors has been evaluated on the scale of different characteristics such as sensitivity, detection limit and repeatability which are required for good performance and life of sensors. Already, different types of graphene sensors have used in medical applications such as measuring glucose level, uric acid, cholesterol level and many more [13].

\section{System design and implementation}

The proposed system was designed to gather psychological signals such as ECG and CBT from the ear and provide a platform to the user for monitoring their health condition with mobile devices in real time. In order to achieve the aims, the system was designed with the capability to extract physiological information from the ear. This section will describe the experimental approach of the study. Figure 1 shows the block diagram of the proposed system.

In order to measure the ECG signal, e-health module kit for Arduino was used. E-health provides health monitoring platform, which has the capability to measure different vital signs. For example, it also allows 3-lead ECG recording using specific pins on the board. The board is compatible with controller board such as Arduino UNO. Figure 2 shows the e-health kit where the controller board is used to extract analogue ECG data from the device which is then converted into digital format using the microcontroller ADC. UART communication protocol is used to send data to the mobile phone for visualization. The Bluetooth module was used for communication purposes.

The Arduino UNO microcontroller board is based on AT mega 328 controller. It provides digital and analogue $\mathrm{I} / \mathrm{O}$, and different communication ports. It consists of $16 \mathrm{MHz}$ crystal oscillator. HC-05 Bluetooth module [14] is used for the wireless transmission of data to smart devices such as laptops, tablets and smartphones. The schematic diagram of the electronic system for the measurement of vital signs is shown in Figure 3. The schematic diagram shows the connection of ECG and CBT sensors on one single board microcontroller.

Figure 4 shows the data acquisition system with integrated sensor along with a Bluetooth module for wireless communication and battery to power up the device. The electrodes are placed behind the ear as shown on the right-hand side of the figure, while the electrical ground connection is connected to the torso.

\section{Results and discussion}

The results obtained from the ECG measurements with different types of electrodes such as $\mathrm{Ag} / \mathrm{AgCl}$ gel type and graphene coated electrodes, and the results compared and discussed. The CBT sensor was inserted into the ear canal (opposite ear - not shown on the figure) and the data recorded is also presented below.

\section{Data recording}

Initially, different tests related to ECG measurements were performed on a commercially available monitoring system (Patient 


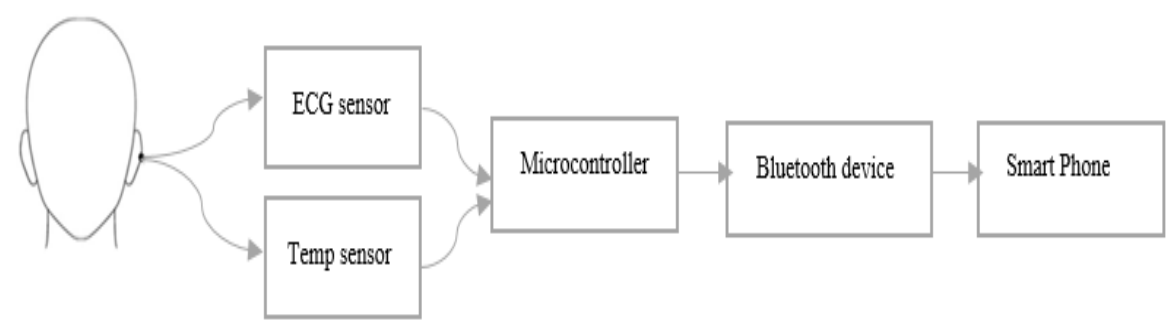

Figure 1. Block diagram of the system.
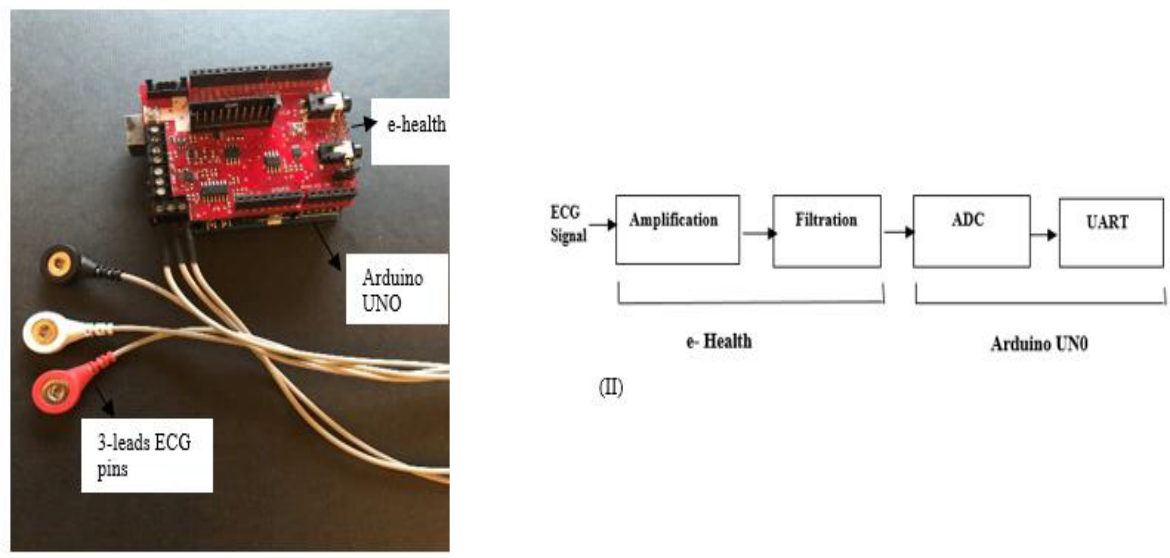

(II)

(I)

Figure 2. E-health kit with Arduino UNO microsystem and data acquisition system.

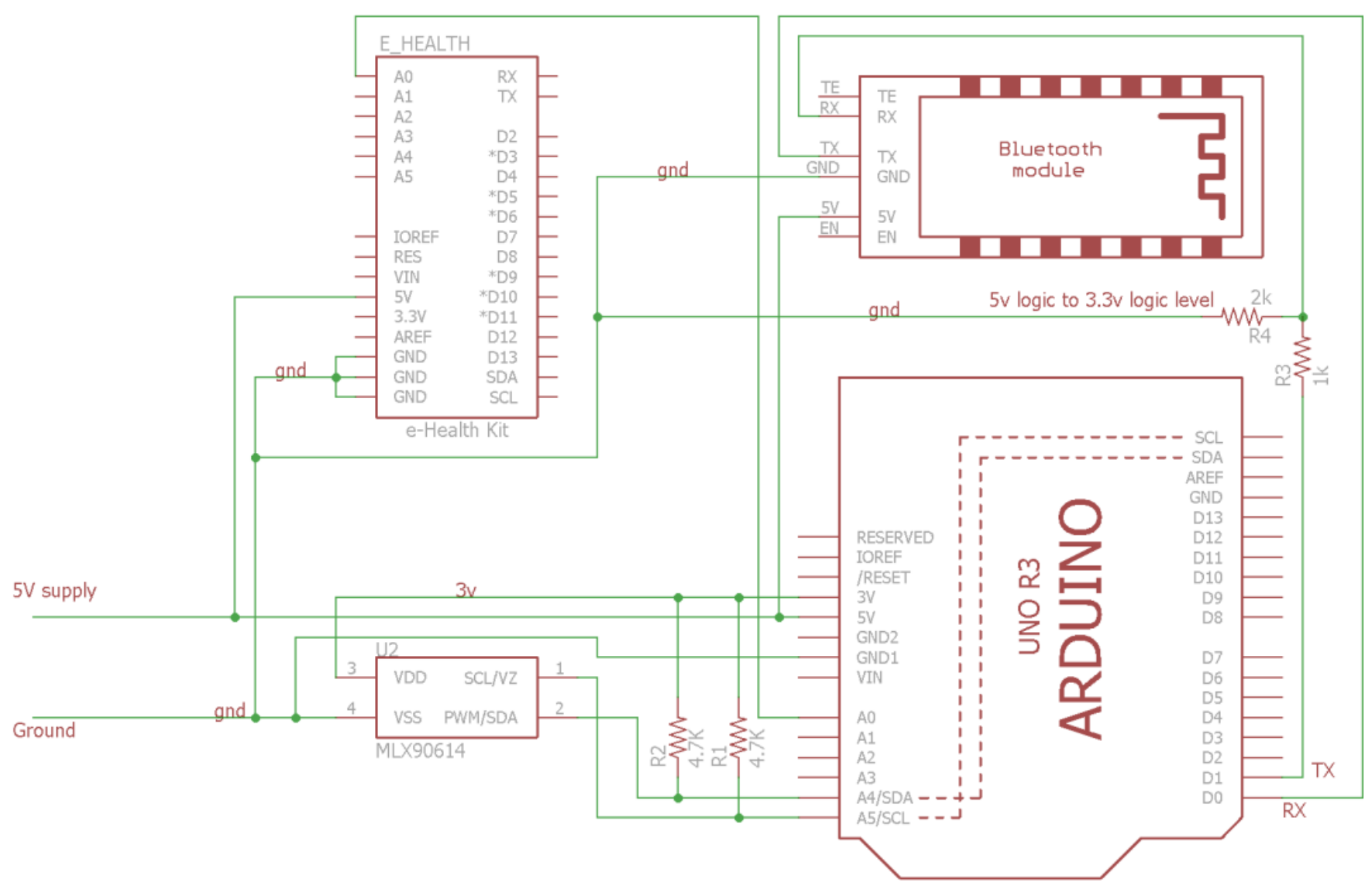

Figure 3. Schematic diagram of the data acquisition system. 


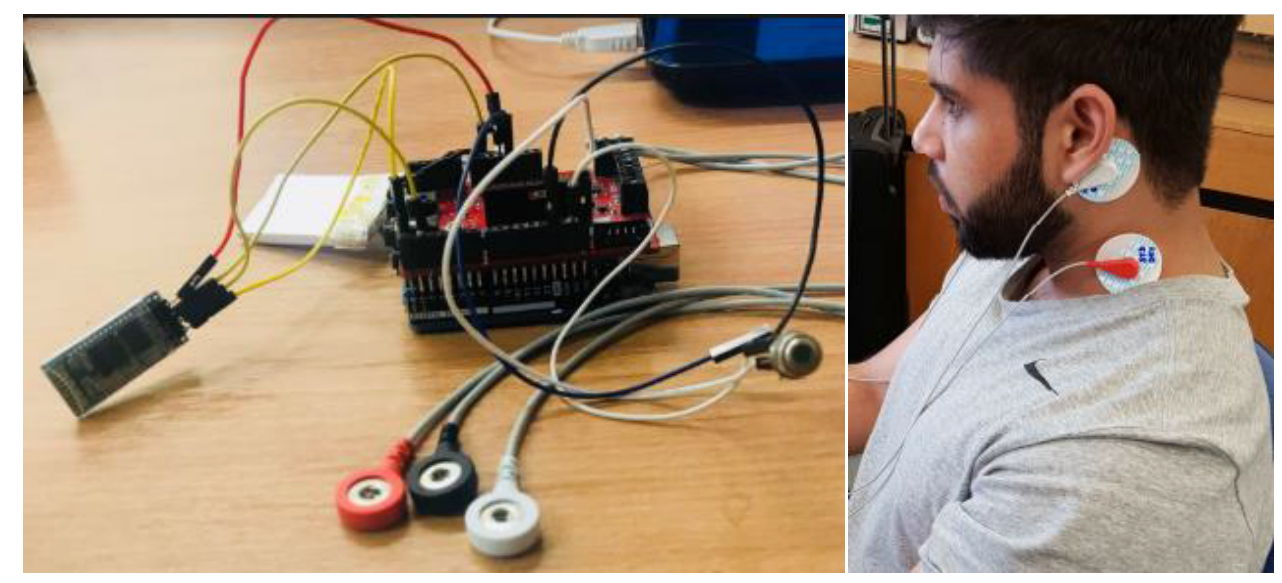

Figure 4. ECG data acquisition system with sensors and Bluetooth device and the electrodes placement behind the ear.

Monitor PM12, Braun international) [15]. It has the capability to measure the different vital signs such as PPG, ECG, CBT and BPM and also provides a graphical user-friendly interface. The initial test was conducted to understand the behaviour of signals with respect to its complexity and also used to determine the correct positions for electrodes placement to extract the ECG signals. Commercially available $\mathrm{Ag} / \mathrm{AgCl}$ electrodes were firstly placed on the chest with the help of the 3-lead technique. Furthermore, the same electrodes were placed behind the ear and the monitoring system was used to investigate the correct placement of electrodes behind the ear with reference to the chest signals.

Two types of electrodes were used to record the signal behind the ear. The 1 st being $\mathrm{Ag} / \mathrm{AgCl}$ gel type, the $2^{\text {nd }}$ is a grapheme-coated electrode. A non-hairy 24-year-old subject without any medical problem was selected in order to perform a different experiment for investigating the extracted signals. The 1st case using type 1 electrode is shown in Figure 5; the highest amplitude of the $\mathrm{R}$ wave is $3500 \mu \mathrm{V}$ using a distance of $8 \mathrm{~cm}$ between the electrodes. The SNR of the signal is $36 \mathrm{~dB}$. However, $\mathrm{P}$ wave of the signal is not clearly visible due to high resistivity between electrodes and skin, but the rest of the critical signs such as QRS complex and T wave are visible.

The $2^{\text {nd }}$ case, the experiment was performed by placing the type 2 electrodes behind the ear with the ground connected to the torso. It can be seen from Figure 6 the fluctuation in signal due to noise is reduced because of better electrical conductivity of the graphene coated electrodes; it provided an improved SNR value of $39 \mathrm{~dB}$. The $P$ wave can be identified and the amplitude of the QRS complex is higher than 3600 $\mu \mathrm{V}$ in this case. It was mentioned above that the graphene provided the good connectivity between the skin and electrodes which helped to obtain the higher amplitude of the wave.

\section{Mobile system testing}

The integrated system was tested using two scenarios; first CBT measurement of temperature and $2^{\text {nd }}$ is the measurements of ECG signal. The CBT measurement was recorded using the graphene coated thermopile sensor, which was inserted close to the tympanic membrane in the ear canal. The device was measuring the temperature with a sampling frequency of $2 \mathrm{~Hz}$. The temperature monitoring was carried out using MATLAB software while the data was gathered with the help of the microcontroller.
The test was conducted in normal condition without any physical activity. As shown in Figure 7, the CBT varies between $36.7^{\circ} \mathrm{C}$ to 37.01 ${ }^{\circ} \mathrm{C}$, which lies in the range of normal CBT of a human being with stable readings.

As for the ECG signal recording, the app running on the phone is capable to receive serial data from the data acquisition device in real time, which further will display on the screen of the phone and allow the user to monitor the ECG signal in user friendly situation. Figure 8 shows the ECG signal from the subject which was taken with graphene coated electrodes. The ECG data from the acquisition unit to the smartphone was sent at $124 \mathrm{~Hz}$ sampling frequency; the data were transmitted to the App using Bluetooth technology.

\section{Conclusions and future work}

In this study, different electrodes placement scenarios were utilized to record and trace the measurable ECG signals from behind the ear to develop the ear-based ECG monitoring device. The comparative test was conducted to analyse the difference between the signals obtained using electrodes behind the ear and chest with the help of both Ag/ $\mathrm{AgCl}$ and graphene-based electrodes. Traditional chest measurement technique provided the best SNR value of about $40 \mathrm{~dB}$ with the help of graphene electrodes, which is higher than that obtained with $\mathrm{Ag}$ / $\mathrm{AgCl}$ gel type electrodes; the value of SNR is about $39 \mathrm{~dB}$. All the critical parameters such as $\mathrm{P}$ wave, $\mathrm{QRS}$ complex and $\mathrm{T}$ waves were visible with less distortion.

For ECG acquisition it was noticed that the behind the ear location has advantages, it provides an easy testing environment with less irritation to the patient and reduces the artefact of the measurement. This proposed system provides the environment to measure both the ECG signal behind the ear and CBT signal from the ear canal using graphene coated electrodes with the required accuracy in a friendly and pain free environment. The CBT measurement was taken from the tympanic membrane inside the ear canal. The tympanic area receives the same blood supply to the hypothalamus; hence the measured CBT value reflects the true core body temperature. The App developed for the smartphone records both ECG and CBT in real time. Future work will focus on improving the surface coating of ECG and CBT sensors with graphene ink by spray coating and it is anticipated that this process will evenly and homogeneously cover the surfaces, which can further reduce the skin electrode impedance as well as improved IR transmission of tympanic membrane temperature more accurately and provide better signals. 


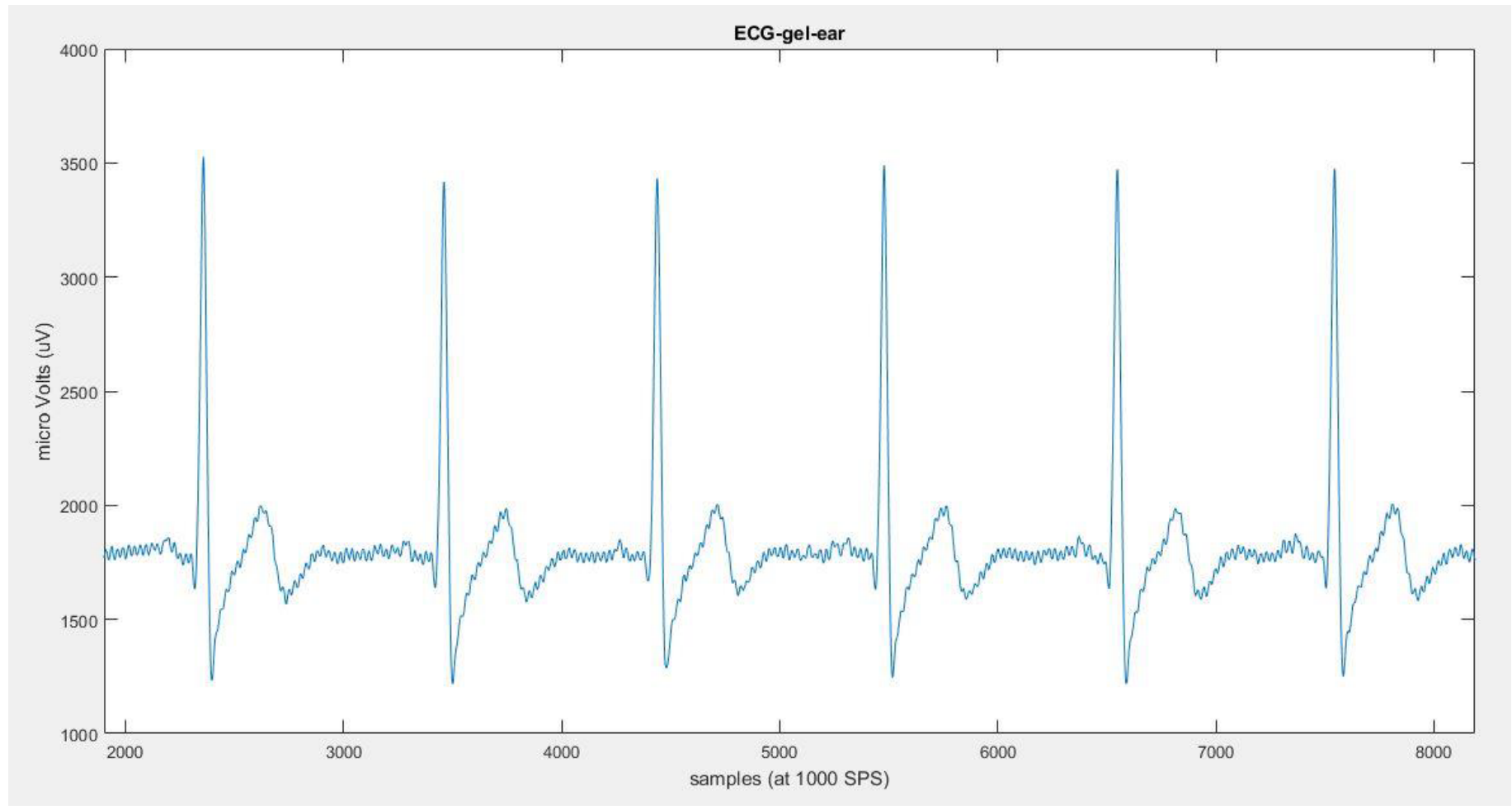

Figure 5. Recorded ECG signal behind the ear using type 1 electrodes.

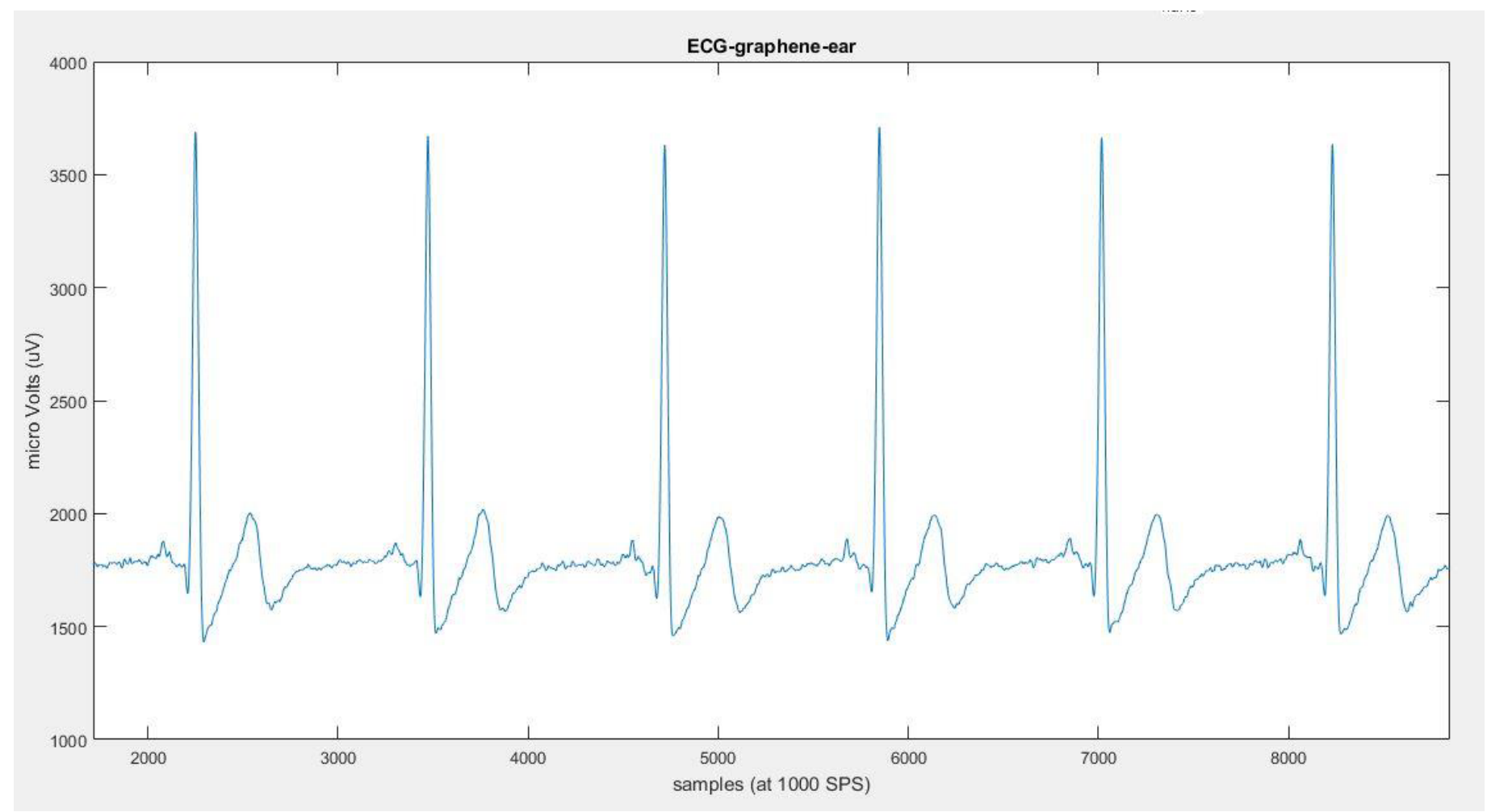

Figure 6. Recorded ECG signal behind the ear using type 2 electrodes. 


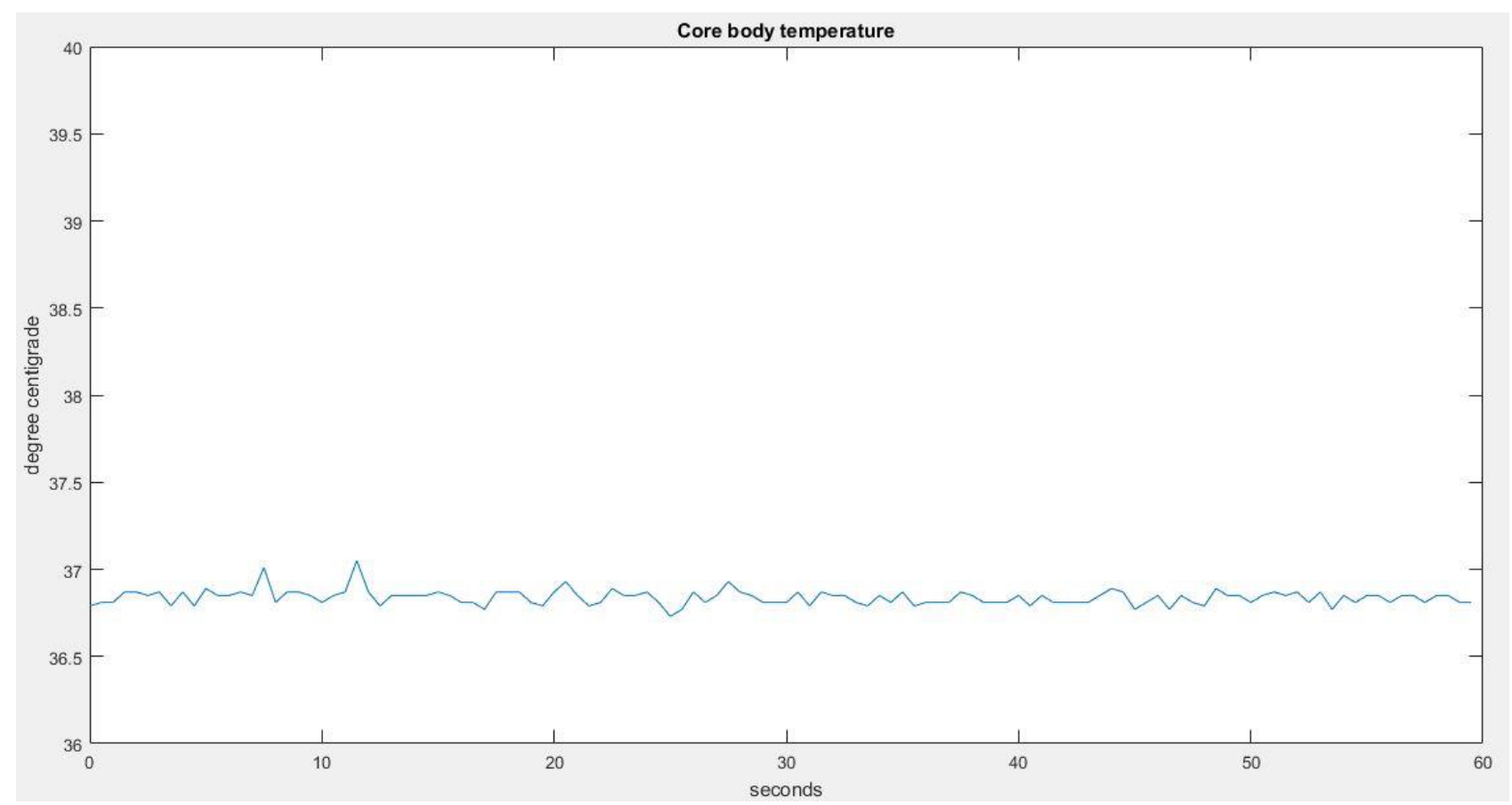

Figure 7. Monitoring of CBT using the mobile device.

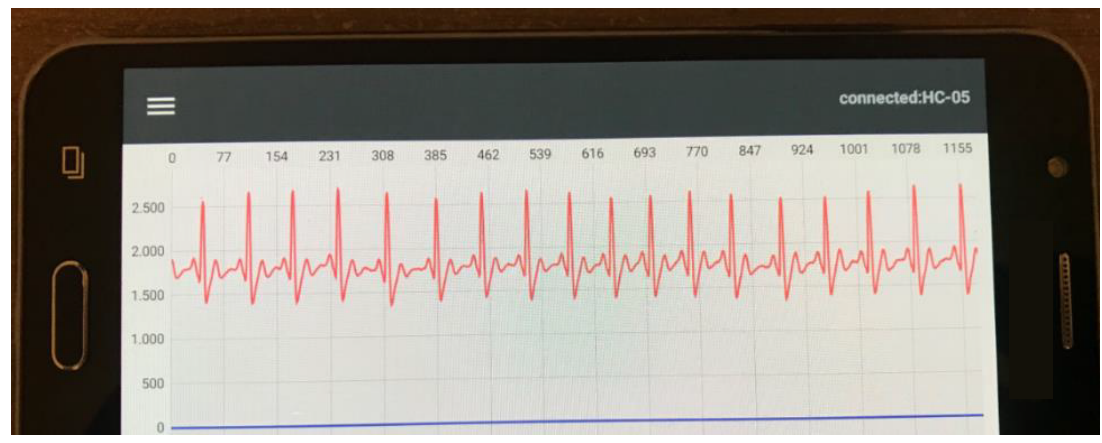

Figure 8. Monitoring of ECG signal on the app running on an Android phone.

\section{References}

1. Martínez-Pérez B, de la Torre-Díez I, López-Coronado M (2013) Mobile Health Applications for the Most Prevalent Conditions by the World Health Organization: Review and Analysis. J Med Internet Res 15: e120. [Crossref]

2. Laslett LJ, Alagona P Jr, Clark BA 3rd, Drozda JP Jr, Saldivar F, et al. (2012) The worldwide environment of cardiovascular disease: prevalence, diagnosis, therapy, and policy issues: a report from the American College of Cardi-ology. J Am Coll Cardiol 60: 25. [Crossref]

3. Jamil KY, Yuce MR (2016) Wireless body area network (WBAN) for medical applications. In New Develop Biomed Eng 2: 99-110

4. He DD, Winokur ES, Sodini CG (2015) An Ear-Worn Vital Signs Monitor. IEEE Trans Biomed Eng 62: 2547-2552. [Crossref]

5. Lee HJ, Lee SH, Ha KS, Jang HC, Chung WY, et al. (2009) Ubiquitous healthcare service using Zigbee and mobile phone for elderly patients. Int J Med Inform 78: 193 198, 2009. [Crossref]

6. Lin YH, Lin CF, You YZ (2011) A driver's physiological monitoring system based on a wearable PPG sensor and a smartphone. Communi Comp Inform Sci 223: 326-335.

7. Mundt C, Montgomery K, Udoh U, Barker V, Thonier G, et al. (2005) A Multiparameter Wearable Physiologic Monitoring System for Space and Terrestrial Applications. IEEE Trans Inf Technol Biomed 9: 382-391.

8. Vescio B, Salsone M, Gambardella A, Quattrone A (2018) Comparison between Electrocardiographic and Earlobe Pulse Photoplethysmographic Detection for Evaluating Heart Rate Variability in Healthy Subjects in Short- and Long-Term Recordings. Sensors (Basel) 18: 844. [Crossref]
9. Poh M, Kim K, Goessling A, Swenson N, Picard R (2012) Cardiovascular Monitoring Using Earphones and a Mobile Device. IEEE Pervasive Comput 11: 18-26.

10. https://www.graphenea.com/pages/graphene-properties\#.W4061-hKjIU

11. Celik N, Manivannan A (2016) Strudwick and W. Balachandran, "Graphene-Enabled Electrodes for Electrocardiogram Monitoring. Nanomaterials (Basel) 6: 156. [Crossref]

12. Justino C, Gomes A, Freitas A, Duarte A, Rocha-Santos T (2017) Gra-phene based sensors and biosensors. TrAC Trends in Analytical Chemis-try91 91: 53-66.

13. Sahatiya P, Puttapati S, Srikanth V, Badhulika S (2016) Graphene-based wearable temperature sensor and infrared photodetector on a flexible polyimide substrate. Flexible Printed Electronics 1: 025006

14. https://hobbycomponents.com/wired-wireless/432-hc-05-master-slave-bluetooth-module

15. http://www.brauninternational.com/shop-home/medical/patient-monitoring/centralmonitoring-systems-detail\#.WM8Ljkue-c8

Copyright: (C2019 Butt M. This is an open-access article distributed under the terms of the Creative Commons Attribution License, which permits unrestricted use, distribution, and reproduction in any medium, provided the original author and source are credited. 